\title{
Predicting the packing behaviour of porous organic cages
}

\section{Emma Helen Wolpert, Kim E Jelfs}

Department of Chemistry, Imperial College London, Molecular Sciences Research Hub, White City Campus, London, W12 0BZ, UK;

\author{
e.wolpert@imperial.ac.uk
}

Porous organic cages are a subset of porous materials which are made up of covalently bonded organic molecules forming cages with intrinsic porosity. Unlike extended framework materials, such as metal organic frameworks which are connected through covalent or coordination bonds, the assembly of porous organic cages is defined by weak dispersion forces. Therefore, the connectivity between the cages can be easily manipulated by varying the chemical functionality or solvent [1]. This leads to a variety of porous organic cage solids which, depending on the packing behaviour, may contain only intrinsic cavities or have extrinsic pores between the cages resulting in one, two, or, three dimensional pore networks [2]. Consequently, the packing behaviour of the porous organic cages can have a vast effect on the properties of the material [3]. It has been suggested that in principle, different cages can be combined to produce structures with specific properties [4]. However, the challenge in reliably predicting the packing behaviour of molecular crystals, due to the lack of strong bonding networks, results in difficulty in targeted design [4].

Although crystal structure prediction can accurately determine crystal energy landscapes, it is computationally expensive to apply to multiple molecular combinations [5]. Here we aim to determine the packing behaviour of porous organic cages through coarse graining. We start by creating a coarse grained Hamiltonian containing the dominant intermolecular interactions between the cages, informed by force field models. We then aim to employ Monte Carlo simulations using our model in conjunction with hard particle Monte Carlo simulations [6] to determine the thermodynamic phase behaviour of the packing of the cages. This work focuses on the well-studied porous organic cage CC3 [1] as a proof-of-concept example to determine the extent to which we can use coarse graining to analyse the packing behaviour of other, less well-studied porous organic cages.

[1] T. Tozawa, J. Jones, S. Swamy, et al. Nature Mater 8, 973-978 (2009)

[2] Y. Liu, G. Zhu, W. You, et al. J. Phys. Chem. C 123, 3, 1720-1729 (2019)

[3] M. E. Briggs and A. I. Cooper Chem. Mater. 29, 1, 149-157 (2017)

[4] J. Jones, T. Hasell, X. Wu, et al. Nature 474, 367-371 (2011)

[5] T. Hasell, S. Y. Chong, K. E. Jelfs, et al. J. Am. Chem. Soc. 134, 1, 588-598 (2012)

[6] J. A. Anderson, M. E. Irrgang, and S. C. Glotzer. Computer Physics Communications 204, 21-30 (2016)

Keywords: Porous organic cages, modelling, coarse graining 